Relations industrielles

Industrial Relations

\title{
Droits individuels et droits collectifs
} Un difficile mais nécessaire appariement !

\section{Fernand Morin}

Volume 64, numéro 1, hiver 2009

URI : https://id.erudit.org/iderudit/029543ar

DOI : https://doi.org/10.7202/029543ar

Aller au sommaire du numéro

Éditeur(s)

Département des relations industrielles de l'Université Laval

ISSN

0034-379X (imprimé)

1703-8138 (numérique)

Découvrir la revue

Citer cet article

Morin, F. (2009). Droits individuels et droits collectifs : un difficile mais nécessaire appariement ! Relations industrielles / Industrial Relations, 64(1), 154-166. https://doi.org/10.7202/029543ar

Tous droits réservés @ C Département des relations industrielles de l'Universite Laval, 2009
Ce document est protégé par la loi sur le droit d'auteur. L'utilisation des services d'Érudit (y compris la reproduction) est assujettie à sa politique d'utilisation que vous pouvez consulter en ligne.

https://apropos.erudit.org/fr/usagers/politique-dutilisation/ 


\title{
DROIT DU TRAVAIL
}

\section{Droits individuels et droits collectifs : un difficile mais nécessaire appariement !}

\author{
$\mathrm{M}^{\mathrm{e}}$ Fernand Morin
}

Pourquoi, comment et jusqu'à quelle limite l'arbitre de grief devrait-il savoir distinguer les droits subjectifs d'un salarié des droits collectifs relevant en propre du syndicat accrédité ? Si le salarié est partie d'un tout (I'unité d'accréditation), ce tout est néanmoins plus, ou autre, que la simple somme des salariés qui le constituent. La situation inversée est aussi vraie, à savoir que la personne du salarié demeure distincte sous de multiples aspects de celle du syndicat. II nous faut aussi reconnaître que ces mêmes distinctions ne sont guère toujours claires et il subsiste effectivement un danger de confusion des genres en raison même de leurs intimes et complexes rapports. Ce glissement, ou ce dérapage conceptuel plus ou moins conscient, est parfois l'effet d'une simple métonymie consistant à prendre la partie pour le tout ou I'inverse, soit le tout pour la partie. Dans le domaine des relations du travail, comme en bien d'autres milieux, nous commettons souvent de semblables confusions langagières. Ne dit-on pas, au sens propre ou figuré, à titre d'exemples, que :

- I'entreprise $X$ est syndiquée !

- Jean-Charles est membre de telle centrale syndicale !

- une centrale syndicale fut accréditée à l'égard de telle entreprise !

- un salarié syndiqué n'a plus d'autonomie juridique en son milieu de travail puisqu'il est subordonné aux consignes syndicales impératives !

- etc.

Ces façons de dire, ces formules elliptiques, ces contractions langagières ou outrancières, ces assertions globales et parfois radicales résultent de sous-entendus, de conventions implicites ou de communes connivences. Souvent, elles sont entretenues ou amplifiées par les médias d'information qui, par recherche de concision, emploient à tort des formules elliptiques mais fausses.

\section{Distinction entre la partie et le tout}

II est vrai que ce rapport " collectif/individu » est souvent complexe et, sous de multiples aspects, insécable. Ce collectif ou ce groupe comprend et est formé de salariés et sans eux, il n'y aurait pas de telle collectivité structurée, constituée et 
agissante. Mais il est tout aussi vrai que sans cette structure organisationnelle, ce faisceau de salariés demeurerait, semblable à l'anémone de mer, sans forme fixe ni couleur propre.

Si des salariés se sont formés en groupes distincts disposant respectivement, à ce titre, de moyens d'expression et d'actions unifiés et cohérents, n'est-ce pas parce qu'ils prirent d'abord conscience, à des degrés divers il est vrai, qu'ils partageaient forcément les mêmes contraintes et, notamment, des besoins et des pressions d'ordre socio-économique ? Face à l'unicité structurelle et d'actions de leur employeur, les salariés sont individuellement d'autant plus faibles qu'ils sont remplaçables les uns par les autres et que chacun est distinctement placé sous l'autorité de la même personne, leur employeur commun. N'est-ce pas en raison de cette même trame socioéconomique étayée par le droit que provient cette nécessité d'établir des réseaux collectifs de rapports du travail ? Par cette dernière voie, on tente de faire ensemble ce que isolément chaque salarié ne peut faire, soit participer à l'élaboration de ses conditions de travail par un réel exercice de sa liberté contractuelle'. Tel est d'ailleurs le sens enfin reconnu par la Cour suprême du Canada de la liberté d'association².

Ces relations collectives du travail, pour être efficientes, exigent de la part des salariés discipline, ordre et cohérence. De semblables qualités ne peuvent s'acquérir que par une prise de conscience et une volonté d'agir ensemble alors que le système juridico-économique confère pareils attributs (discipline, ordre et cohérence) à l'entrepreneur et ce, dès qu'il le devient, et de ce seul fait ${ }^{3}$. Pour cette première raison, on ne saurait croire, imposer ou juger tous les actes d'un salarié à l'aide des mêmes critères d'évaluation que ceux applicables à leur employeur, lequel a pu et peut encore exercer sa liberté contractuelle. Les contraintes et les libertés respectives des salariés sont à ce point différentes de celles de l'employeur qu'il leur faut acquérir ensemble une « égalité »fonctionnelle pour pouvoir exercer par ce biais collectif leur liberté contractuelle.

Notre première question consiste à savoir en quoi, pourquoi et quand un groupe de salariés, ce collectif, se distingue de chacun de ses membres qui le forment, puis comment assurer valablement ce partage des droits collectifs de ceux propres à chacun des salariés qui composent ce même groupe ? La question demeure importante et essentielle parce qu'on peut parfois oublier, dans le cadre de la discussion, qu'un salarié, membre d'un syndicat, demeure néanmoins et toujours une personne, un citoyen à part entière, un titulaire de droits et un justiciable. Par ailleurs, il est tout aussi vrai, en fait et en droit, qu'une collectivité de salariés constituant une unité d'accréditation, au sens et pour les fins du Code du travail (Ct), se distingue de chacun des salariés qui la forment. À titre d'illustration, considérons le comportement d'un groupe quelconque de personnes par rapport à celui de chacun de ses membres compris isolément les uns des autres. Leur manière d'agir et de réagir est fort différente selon qu'il s'agisse d'un salarié isolé ou d'un groupe de salariés constitué en unité d'accréditation et représenté à ce titre par un syndicat accrédité. Notre commune expérience ne nous enseigne-t-elle pas que la dynamique ou la psychologie d'un groupe n'est nullement comparable à celle de chaque individu qui s'y retrouve : 
- un étudiant seul et une classe d'étudiants !

- un député seul en son comté ou les députés réunis à l'Assemblée nationale !

- un amateur de hockey seul et un groupe d'amateurs à leur sortie suite à une magistrale victoire ou défaite!

- un salarié qui discute avec son contremaître et un groupe de salariés qui refusent d'obtempérer à une consigne patronale !

- etc.

Pour ces premières raisons, on ne saurait valablement apprécier ou juger les actes d'un groupe par simple voie inductive où la personne dite raisonnable, réfléchie et prudente, servirait de modèle. II y a lieu, en certains cas ou sous certains aspects, de distinguer le tout de ses parties qui s'y retrouvent ${ }^{4}$. Si, de fait, une collectivité de salariés est différente de chacun des salariés qui la composent, cette même collectivité ne peut masquer chacun de ces mêmes salariés sous tous les aspects des relations du travail. Notons que la Cour suprême du Canada sut faire cette dernière distinction lorsqu'un syndicat entendait refuser un accommodement raisonnable à un salarié prétextant que telle était la volonté générale de ses membres 5 .

Pour mieux illustrer cette nécessaire distinction entre les salariés et leur syndicat, considérons leur notion respective du temps pour en tirer un enseignement utile. Chacun des salariés du groupe assume lui-même sa vie professionnelle au jour le jour et ses besoins personnels et familiaux immédiats constituent son premier souci alors que le syndicat peut faire une tout autre lecture du temps. C'est ainsi que chaque négociation collective, chaque traitement d'un grief, prépare, aménage ou influe sur le traitement futur des autres négociations collectives ou règlements de grief. Combien de conditions de travail actuelles arrêtées à une même convention collective sont le résultat cumulatif de deux ou de trois négociations collectives antérieures, c'est-àdire un travail collectif étalé sur dix ans ? Le comportement du salarié peut être fort différent, notamment s'il éprouve un besoin immédiat de liquidité : ne pourrait-il pas alors fort bien accepter de travailler un samedi à taux simple ? II nous faut croire que le syndicat s'y opposerait parce que le précédent ainsi constitué serait préjudiciable à tous ses collègues de travail et rendrait difficile la prochaine négociation sur ce même point. C'est ainsi, et en bien d'autres situations, que les effets du « temps court » d'un salarié diffèrent sensiblement de la portée du « temps long » du syndicat.

N'est-ce pas en fonction de ce même impératif que l'article 69 du Code du travail ${ }^{6}$ reconnaît qu'un syndicat puisse faire un grief en faveur du respect des droits d'un salarié sans obtenir préalablement un mandat spécifique de la part de ce dernier, et même de pouvoir le faire alors que ce salarié s'y opposerait ? Grâce à cette distinction des droits individuels et collectifs, ceux du syndicat et ceux du salarié, ce dernier demeure ainsi protégé par la structure syndicale sans personnellement devoir se commettre auprès de son employeur alors qu'à ce niveau, il demeure fort vulnérable. Ainsi, les actes que poserait autrement un salarié en raison d'un aveuglement passager (effet du temps court) ne peuvent préjudicier l'ensemble de ses collègues. Cet actuel article 69 du Code du travail résulte d'un enseignement pratique retenu par le législateur, tout comme 
en témoigne aussi maintenant les mesures protectrices du salarié que I'on retrouve aux articles 2089, alinéa 3, 2092, 2095 et 2097 du Code civil du Québec (CcQ). On ne saurait donc être surpris que certains actes rattachés aux rapports collectifs du travail (statut du syndicat, accréditation, négociation collective, convention collective, arrêt concerté du travail, etc.) soient parfois en conflit ou opposés ou à finalités contraires ou divergentes de ceux relevant de la seule compétence du salarié/individu et, dès lors, que les règles de droit applicables aux individus soient distinctes de celles qui relèvent du syndicat qui le représente auprès de l'employeur. Ces distinctions qu'imposent les impératifs de l'action collective n'exigent ni ne justifient cependant la négation des droits du salarié qui lui résultent de sources autres que la convention collective.

\section{De la mixité des sources de droit}

S'il en est ainsi, et ce depuis longtemps, pourquoi la question du départage ou de I'appariement des droits collectifs et des droits individuels serait-elle aujourd'hui plus importante ou se présenterait-elle avec plus d'acuité ? Les conflits de droits entre ceux relevant de la personne du salarié/individu et ceux qui sont le propre du syndicat à titre de représentant du collectif des salariés surviennent maintenant beaucoup plus fréquemment du fait :

- d'une application plus certaine ou plus généralisée des droits et des libertés garantis aux chartes à la personne même du salarié;

- de la diversité des sources génératrices de normes du travail; et, aussi,

- d'une jurisprudence parfois malaisée à circonscrire en raison même de sa facture hétérogène ${ }^{7}$.

Sans égard ou par-delà la conclusion d'une convention collective, le salarié, à titre d'individu, dispose d'un faisceau de droits et de libertés spécifiques qui ne peuvent ou ne devraient pas être écartés, niés, altérés, modulés ou bémolisés par la convention collective. Telle est la base principale de notre thèse en cette problématique. Soulignons qu'un tel impératif n'est pas le fruit d'une visée prétorienne, ni une simple théorie personnelle puisqu'elle repose notamment sur le principe de la hiérarchie des normes ${ }^{8}$. D'ailleurs, ce même principe n'est-il pas expressément retenu à l'article 62 du Code du travail, lequel délimite la liberté des parties à la convention collective en précisant que cette liberté conventionnelle est circonscrite par les règles de droit d'ordre public et des prohibitions édictées par les lois. Au nombre de ces règles de droit, il nous faut d'abord retenir le contenu des chartes qui affirment l'égalité des citoyens et garantissent leur liberté de conscience, de religion, d'opinion, d'expression, de réunion et d'association. À cette première et essentielle catégorie de droits individuels s'ajoutent les droits que leur confèrent les lois sur l'emploi (Loi sur les normes du travail [LNT], Loi sur les accidents du travail et les maladies professionnelles [LATMP], Loi sur la santé et la sécurité du travail [LSST], Loi sur la protection des renseignements personnels [LPRP], etc.). Ces lois édictent des conditions de travail spécifiques applicables à tout un chacun des salariés qui, en raison de leur caractère d'ordre public, ne peuvent davantage subir quelques grignotages par le truchement 
d'une convention collective suite à un quelconque troc. En d'autres termes, les parties à la convention collective disposent d'un espace juridique libre que leur laissent les lois et ces parties ne peuvent, même ensemble, substituer leur convention à ces lois ${ }^{9}$. Telle est du moins notre thèse ${ }^{10}$.

Bien que les chartes furent promulguées il y a plus de vingt-cinq ans, la transposition pratique de leur contenu demeure assez lente, malgré qu'elle connaisse une certaine croissance. À titre d'exemple, il fallut vingt ans avant que la Cour suprême du Canada reconnaisse que la liberté d'association affirmée à la Charte canadienne (art. 2, al. d) se traduisait bien, pour les salariés, en une liberté de tenir des rapports collectifs avec leur employeur, que ce dernier se devait de négocier de bonne foi et qu'un syndicat n'avait pas la même finalité historique et pratique qu'une association de joueurs d'échecs ${ }^{11}$. Aussi, le traitement médiatique des séances publiques tenues par la Commission Taylor-Bouchard eut l'effet de sensibiliser bien des salariés à ce moyen particulier de correction appelé « accommodement raisonnable $»^{12}$. Ainsi, peu à peu, les salariés apprennent qu'ils ont des droits et des libertés, et que ni l'employeur ni leur propre syndicat ne peuvent valablement y porter atteinte. De telles situations constituent autant de foyers à d'éventuels conflits de droits individuels ou collectifs qui peuvent être maintenant évoqués ou invoqués par l'une ou l'autre des personnes intéressées à l'occasion d'un grief. En pareil contexte, il incombe alors à l'arbitre de départager ou d'apparier et il doit le faire en sachant justifier sa décision autrement que par de simples renvois (la formule « perroquet ») aux dits des tribunaux judiciaires.

Outre cette première et essentielle prise de conscience des libertés affirmées, le droit substantif de l'emploi s'est complexifié de multiples façons au cours des dernières décennies. Ce n'est que depuis vingt-cinq ans et parfois fort plus récemment qu'un salarié dispose :

- du droit de refus d'obtempérer à une directive de l'employeur, s'il a raison de croire que l'exécution de la tâche demandée l'exposerait à un danger pour sa santé, sa sécurité ou son intégrité (art. 12 LSST);

- du droit de participer à la mise en place d'un régime adapté de sécurité au travail dans l'entreprise qui l'occupe et de disposer, à cette fin, de moyens nécessaires à sa propre sécurité et à la sauvegarde de sa dignité (art. 2, 9 et 10 LSST);

- du droit de l'accidenté du travail de conserver en continu, au cours de son absence, son ancienneté au sens et pour les fins de la convention collective (art. 235, LATMP) et de disposer d'un droit à la réintégration de son emploi au terme de cette absence (art. 236 et s. LATMP);

- du droit de faire légalement grève sans qu'une tierce personne puisse occuper valablement son poste (art. 109.1 Ct) et sachant qu'il pourra le recouvrer dès le retour au travail convenu (art. 110.1 Ct);

- du droit du salarié congédié d'imposer un contrôle de la qualité de la représentation du syndicat accrédité à cette occasion auprès de l'employeur (art. 47.2 et s. Ct); 
- du droit de refus d'une prise de retraite au moment fixé par l'employeur ou reconnu à la convention collective à titre d'âge «normal » de retraite (art. 84.1 LNT);

- du droit au remboursement de ses frais de déplacement ou de formation lorsque de telles activités sont requises par l'employeur (art. 85.2 LNT);

- du droit au maintien de son emploi suite à une absence pour maladie ou un accident, de moins de vingt-sept semaines s'il dispose déjà de trois mois de service continu (art. 79.1 LNT);

- du droit d'exercer un recours par voie de grief s'il a raison de croire qu'il est victime d'un harcèlement psychologique (art. 81.20 LNT);

- etc.

Depuis 1994, le Code civil du Québec reconnaît le particularisme du contrat de travail fondé notamment sur la situation de subordination juridique du salarié et ce, en vue de lui conférer des mesures de protection inaliénables (art. 2087, 2089, 2095 et 2097 (cQ) ${ }^{13}$.

Ces multiples et nécessaires interventions législatives peuvent néanmoins provoquer certains conflits de droits réels ou apparents, résultant notamment d'inévitables interrelations fonctionnelles des lois et des conventions collectives. II suffit de considérer concurremment le libellé de maintes dispositions des lois sur I'emploi et celui de plusieurs conventions collectives pour constater les multiples liens de complémentarité ou de chevauchement des conditions de travail aménagées par la voie combinée de ces deux sources.

Si les conventions collectives contiennent des conditions de travail adaptées à leur milieu respectif, certaines ne sont que de simples prolongements ou des modulations de dispositions législatives et alors elles doivent être formulées en fonction du cadre fixé par ces dernières (art. $62 \mathrm{Ct}$ ). En d'autres situations, la loi établit presque exclusivement la condition de travail portant sur un objet particulier et qui supplée ainsi à certaines lacunes des conventions collectives ou aux faiblesses d'une partie. II suffirait de considérer les données épistémologiques de plusieurs articles des lois sur l'emploi pour s'en convaincre. À titre d'exemples, nous pourrions rappeler les motifs du législateur lorsqu'il édicta :

- I'article 12 LSST (droit de refus);

- les articles 47 et 63 Ct (sécurité syndicale);

- I'article 81.20 LSST (harcèlement);

- I'article 59.1 LNT (jours fériés);

- I'article 87.1 LNT (clause orphelin);

- etc.

Parfois, le législateur se saisit de la question quasi dans sa totalité soit parce que l'expérience démontra que les parties aux conventions collectives $n$ 'en traitaient guère ou d'une façon insatisfaisante pour les salariés. En d'autres situations, le législateur 
se limite à ouvrir une brèche en énonçant quelques paramètres servant de principes généraux, puis il laisse aux parties le soin de parfaire ce travail de fondation (par ex., art. 59.1 LNT, jours fériés et art. 244 LATMP, retour de l'accidenté). D'autres interventions législatives visent à corriger certains abus des parties à la convention collective. N'estce pas l'effet recherché, soit celui d'enrayer les « clauses orphelins » (art. 87.1 LNT) et soit celui d'imposer un juste calibrage par la Loi sur l'équité salariale, etc. ?

Il en résulte qu'un arbitre ne saurait connaître la réelle portée de bien des conditions de travail applicables dans un milieu de travail sans prendre en compte à la fois l'ensemble des données pertinentes provenant de ces sources complémentaires les unes aux autres et dont les règles ne s'imbriquent pas toujours selon un même ordre linéaire : chartes, Code civil, lois et conventions collectives, etc.

\section{Fonctions et réactions des parties et de l'arbitre}

Dans le contexte particulier d'une négociation collective, il est possible que les parties retiennent certaines dispositions ou qu'elles emploient des formules qui répondent d'abord à leurs principales préoccupations, partagées ou non, et que ce faisant, elles négligent plus ou moins consciemment de tenir compte de la portée directe ou indirecte de quelques règles de droit rattachées au statut même de la personne du salarié. Quoiqu'il en soit des causes occasionnelles à l'origine de semblables situations, nous rappelons que le seul fait que le syndicat soit cosignataire de cet acte juridique ne saurait constituer un facteur de bonification ni de purification de ces entorses ${ }^{14}$.

Pour ces raisons, il est nécessaire que l'arbitre sache apprécier lui-même l'ensemble de la problématique juridique dont il est saisi et qu'il ne se limite pas uniquement ou aveuglement au seul libellé de la convention collective ou à l'entendement des parties au sujet de la réelle portée du grief. C'est aussi dans ce contexte, nous semble-t-il, que la théorie dite « du comptoir unique » trouve sa justification et qu'elle peut être mieux comprise par les tribunaux, les arbitres et les parties aux conventions collectives.

En de telles situations de conflit de droits, l'arbitre de grief doit s'imprégner de cette mixité des sources ou de cette complémentarité multilatérale des règles de droit concernant les conditions de travail des salariés. D'ailleurs, cette préoccupation s'imposerait en raison même de l'économie du régime de justice applicable à ces parties et énoncé clairement à l'article 100 du Code du travail. Ce régime répond d'une logique pratique qui consiste à confier l'ensemble des facettes juridiques d'une même affaire que peut comprendre un grief au seul forum retenu impérativement pour « rendre justice » aux parties à la convention collective. En ce sens, I'arbitrage de grief constitue une véritable « justice de proximité $»^{15}$.

Si certains juges, arbitres ou praticiens doutent de la compétence personnelle des arbitres à pouvoir embrasser aussi grand, demandons-nous quelle serait la différence à effectuer entre la personne d'un arbitre dont la compétence professionnelle spécialisée fut d'abord reconnue par les représentants des centrales syndicales et du patronat (le Conseil consultatif du travail et de la main-d'œuvre) et celle d'un juge de la Cour supérieure qui, avant sa nomination, put exercer dans des champs fort loin du 
droit du travail| ${ }^{16}$. Pourtant, peu de personnes soulèvent pareille question relative à la compétence de ce dernier !

Plusieurs droits individuels que confèrent les lois sur l'emploi donnent ouverture à un recours spécifique et parfois distinct de la voie de l'arbitrage de grief. II est aussi possible que de tels recours soient entrepris avant que l'arbitre soit saisi d'un grief qui porte également sur les mêmes faits. En d'autres termes, une partie ou un salarié peut vouloir exercer ces autres recours concurremment au grief. C'est alors que la question de la compétence juridictionnelle de l'arbitre de grief et de ses limites peut être soulevée et discutée. À ce sujet, nous référons à l'arrêt Concordia que nous avons déjà commenté bien que tous, juges et praticiens, n'en dégagent pas les mêmes réponses ${ }^{17}$.

À l'égard de chaque grief, l'employeur et le syndicat doivent respectivement et aussi concurremment tenir compte des intérêts juridiques propres du salarié. En ce sens, les parties ne peuvent éluder les droits subjectifs dont dispose le salarié en vertu d'une autre source que celle de la convention collective ${ }^{18}$. II en résulte, par voie de conséquence, que l'arbitre doit, au besoin, s'assurer qu'il en est bien ainsi, quelles que soient les prétentions respectives ou partagées des parties ${ }^{19}$. Certes, ces parties ont pu ou ont dû tenir compte de la primauté des droits subjectifs du salarié qui lui résultent de la loi (art. 62, Ct), à l'occasion de l'élaboration de la convention collective et de son administration, mais il convient que l'arbitre s'en assure, d'autant plus qu'un tel entendement peut n'être pas partagé par tous. Certains procureurs justifient la primauté qu'ils accordent à une disposition de la convention collective, même si elle contrevient de quelque manière à un droit que la loi confère à un salarié, en alléguant que, dans l'ensemble, cette convention collective serait plus généreuse que ce que les lois procurent à ce même salarié. À notre avis, une telle « comptabilité » globale ou l'approche dite du « bottom line » ne serait pas valable et, d'ailleurs, elle fut déjà expressément réfutée par la Cour suprême du Canada ${ }^{20}$.

Bien que ce bref rappel des droits récents que confèrent les lois du travail au salarié ne soit nullement exhaustif, ces diverses situations sont autant d'illustrations de situations où l'arbitre peut devoir s'assurer que tous les droits du salarié rattachés au grief sont aussi respectés à l'occasion d'un arbitrage entre les parties. L'ignorance, I'inadvertance ou la collusion des parties ne sauraient justifier l'aveuglement conscient ou non de l'arbitre. S'il se doit de s'enquérir du respect de ces règles, il est possible que son intervention soit mal perçue par les parties ou qu'elle perturbe leur plan de travail. Cette situation, parfois inévitable bien que délicate et nullement agréable, n'est pas le fait personnel de l'arbitre et elle demeure un impondérable directement rattaché à l'exercice de sa fonction, laquelle dépasse toujours sa seule personne ${ }^{21}$.

\section{Face à ces différentes situations, que peut faire l'arbitre de grief ?}

Avant d'élaborer un " plan d'action » ou de correction, l'arbitre devrait d'abord bien circonscrire la problématique dont il est saisi afin d'être en position d'indiquer, s'il y a encore lieu, aux procureurs ses premières appréhensions. Ce travail préparatoire pour réaliser cette étape initiale devrait permettre à l'arbitre de s'assurer qu'un tel conflit de 
droits existe vraiment et, dès lors, de jauger sa réelle ampleur. Cet exposé préalable présenté aux procureurs devrait être formulé d'une manière la plus objective possible, car il incombe à ces derniers de prendre eux-mêmes position en fonction des réelles composantes de la situation et des intérêts de leur mandant respectif. Mais, de telles considérations, aussi nécessaires soient-elles, ne diminuent en rien les obligations professionnelles qui relèvent, au premier chef, de l'arbitre valablement saisi d'un grief.

Une fois sensibilisés ou confrontés à nouveau à ce dilemme collectif/individuel par l'arbitre, ces procureurs sont invités à préciser leur position respective et surtout à indiquer la ou les voies de solution qu'ils retiennent, préconisent ou proposent. Si ces propositions communes ou distinctes ne permettent pas de résoudre d'une façon adéquate la question à l'égard des droits particuliers du ou des salariés directement ou indirectement visés et ce, au jugement de l'arbitre, ce dernier devrait en informer les procureurs. À ce sujet, nous croyons que l'arbitre ne serait aucunement lié par une voie de solution retenue par les parties si celle-ci implique, ni plus ni moins, une négation de droits fondamentaux ou d'ordre public d'un salarié. Bien que nommé par les parties, leur entente sur pareille question relative au respect des droits fondamentaux et d'ordre public ne constitue pas, de ce seul fait, un quelconque blanc-seing autorisant ou justifiant l'arbitre à retenir la voie proposée. Notons que l'article 100.2 du Code du travail précise bien que l'arbitre est le « maître » de la procédure et du mode de preuve, à défaut de la convention collective d'y pourvoir (art. 100, al. 3 Ct) ${ }^{22}$. Un tel pouvoir comprend aussi une part de responsabilité qui incombe à l'arbitre.

En l'absence d'une proposition de solution idoine présentée par les procureurs, il revient à l'arbitre d'établir la voie la plus harmonieuse à l'égard de l'ensemble des droits en présence et susceptible de mieux assurer le respect intégral des droits de tous les intéressés dans le cadre et les limites du processus arbitral. Dans le cas d'une situation difficile ou délicate, notamment lorsqu'un des procureurs précise à l'avance qu'il s'opposerait catégoriquement à toute autre voie que pourrait retenir l'arbitre, nous croyons que ce dernier devrait :

- profiter de ce temps d'arrêt pour fin d'écriture d'une décision interlocutoire de manière à mieux soupeser les composantes de sa propre prise de position et de mieux étayer ses conclusions;

- prendre formellement position dans le cadre d'une décision interlocutoire dans laquelle il circonscrit la question, indique les positions des parties, puis justifie la voie retenue pour les seules fins de l'exercice de sa compétence juridictionnelle concernant le grief dont il est saisi et sans plus.

II va de soi qu'une telle sentence interlocutoire oblige les procureurs et les parties à mieux évaluer ou réévaluer à leur tour leur position respective et à délimiter d'autant l'éventuel ou possible débat qui pourrait s'ensuivre au cours du processus arbitral ou en un autre forum.

Outre cette première trame, il est aussi possible que les deux parties reconnaissent, à des degrés variés cependant, que des droits fondamentaux d'un autre salarié ou d'un cadre seraient aussi en cause à l'occasion du traitement du grief qui les occupe. 
Quoiqu'il en soit, un des procureurs ou les deux, peuvent s'opposer à la présence de ce tiers au débat. Certes, peut-on comprendre cette première réaction compte tenu de l'économie générale de notre régime d'enquête judiciaire fondé principalement sur le bipartisme (le débat contradictoire et non le processus inquisitoire). La gêne, la complexité et la lourdeur appréhendées provenant d'un apparent tripartisme peuvent expliquer cette opposition, sans nécessairement la justifier, en tous les cas.

Indirectement ou incidemment, la problématique collectif/individuel peutégalement déboucher sur la question du devoir d'accommodement. II s'agit notamment des situations où l'application d'une règle ou norme « collective » imposerait à un salarié une contrainte, un comportement ou un acte susceptible d'affecter, de limiter ou de porter atteinte à l'exercice d'une liberté fondamentale. En de semblables circonstances, l'arbitre pourrait-il refuser d'intervenir sous prétexte qu'il n'y aurait pas de violation directe de la convention collective ? Notons que l'accord des parties à l'effet que la règle collective ne saurait souffrir d'une quelconque exception ou assouplissement ou que l'assemblée générale des syndiqués s'y refuserait ne peuvent, de ce seul fait et automatiquement, autoriser l'arbitre à s'en satisfaire.

En toutes ces situations, l'arbitre doit exercer pleinement sa " compétence juridictionnelle », que cela plaise ou pas à l'une ou aux deux parties. Si l'arbitre est désigné par ces dernières et même rémunéré par elles, il doit d'abord et avant tout "rendre justice», fonction qui lui est dévolue en vertu du Code du travail et il doit le faire à l'aide des pouvoirs qui lui échoient à ce dernier titre. S'il dispose du pouvoir de dire le droit (juris-dictum), il se doit de le faire envers tous les justiciables visés par le grief ce qui comprend, au premier chef, le salarié23. Parfois, il peut être prudent et même utile de rappeler aux parties la nature de la fonction qu'exerce l'arbitre, car certains, éblouis par des intérêts immédiats, peuvent croire ou laisser entendre que «leur » arbitre est bien à « leur » service exclusif entendu au sens servile du terme ${ }^{24}$.

\section{En guise de conclusion}

Tenant compte de ce faisceau de facteurs impondérables:

- la transposition pratique et mieux adaptée des garanties formelles conférées aux salariés par la Charte et le Code civil du Québec;

- I'obtention par les lois sur l'emploi de droits formulés de manière à mieux protéger le salarié et aussi pouvant servir d'étaiements juridiques à l'une ou à l'autre partie à la convention collective;

- la présence concomitante de multiples voies de recours et d'instances compétentes en vue d'assurer l'application de différents droits du salarié;

- la portée plus ou moins certaine d'arrêts de la Cour suprême du Canada en raison de certains critères de jugement retenus et susceptibles parfois de renverser l'ordre hiérarchique des normes.

De cette mixité de règles de droit, il en résulte parfois une complexité pratique de la trame juridique sur laquelle repose l'exercice de la fonction de l'arbitre de grief. II 
est vrai que ce même ressentiment est partagé par bien des parties aux conventions collectives.

Quoiqu'il en soit de ces difficultés pratiques, si la protection juridique des salariés provient d'une combinaison des lois et des conventions collectives et si l'employeur et le syndicat doivent ensemble convenir de conditions de travail dans le respect intégral des lois (art. 62 Ct), il va de soi, nous semble-t-il, que l'arbitre doit aussi apprendre à respecter et à sauvegarder à la fois l'ensemble des droits dont dispose le salarié, lequel demeure toujours un citoyen à part entière et distinct, à ce titre, du syndicat. L'institution des rapports collectifs du travail n'implique ni n'emporte un communautarisme outrancier.

En d'autres termes, I'arbitre ne saurait se limiter au seul libellé de la convention collective et compris selon le seul entendement conjoint ou distinct des parties. II lui faut saisir les effets combinatoires des règles de droit applicables et cela, même au risque de déplaire à l'une ou aux deux parties directement visées. Le fait, hypothétique il va de soi, que ces dernières s'entendraient pour ignorer ou pour contourner une règle de droit d'ordre public ne peut autoriser ni justifier l'arbitre à faire de même : error communis non facit jus. L'arbitre n'est pas, en ce sens, au « service » des parties; il n'est que désigné par elles et il exerce une fonction, celle de rendre justice, qui, à ces fins, le dépasse, tout comme elle surplombe les parties. Il est certes possible que certains arbitres considèrent ce " service professionnel » ainsi exercé fort délicat ou pénible et aux effets financiers désastreux pour eux, mais tout arbitre doit apprendre, comme en bien d'autres professions, à braver le « feu » réel ou seulement « déclaré » par les uns ou simplement appréhendé par les autres !

\section{Notes}

1 L'exercice d'une liberté sans une certaine égalité à l'égard du vis-à-vis ne peut être qu'éphémère. Ce préalable, l'égalité, s'acquiert par l'adhésion de chacun à un même liant, le syndicat, lequel assure la nécessaire cohésion de l'action commune qu'il permet.

2 L'arrêt Health Services and Support - Facilities Subsector Bargaining Assn. c. ColombieBritannique, [2007] 2 R.C.S. 391, 2007 CSC 27 [Health]. Nous disons « enfin » parce qu'il fallut vingt ans avant que la Cour suprême du Canada constate, en droit, cette même réalité : voir F. Morin, "La tenue des rapports collectifs du travail : un privilège ou un droit ? », Fragments sur l'essentiel du droit de l'emploi (fragment T), Montréal, Wilson et Lafleur, 2007.

3 Si l'entrepreneur est une personne morale, c'est cette personne (la compagnie) qui agit et ses actionnaires demeurent à l'abri de toute responsabilité des faits et gestes de cette dernière, sauf leur mise de fonds respective qui peut en être affectée : bonus, malus.

4 On sut le faire au Code du travail en reconnaissant qu'un arrêt collectif de travail (la grève) ne constituait pas, de ce seul fait, une cause de rupture des contrats de travail (art. $110 \mathrm{Ct}$ ). En somme, la règle du non adimpleti contractus devient alors inapplicable en cette situation où l'acte est réputé celui du groupe et non celui de chacun des salariés et qui pourtant firent grève.

5 Cette "volonté générale » ne pouvait servir d'argument simplement formel. II en serait autrement si ce même refus syndical était fondé sur des motifs sérieux et bien établis, c'est-à-dire que l'accommodement proposé causerait de graves préjudices à certains autres 
salariés: Central Okanagan School District No. 23 c. Renaud, [1992] 2 R.C.S. 970 et notre commentaire dans RI/IR, 48 (4), 1993, 732.

6 Cet article 69 Ct est libellé ainsi : "L'association accréditée peut exercer tous les recours que la convention collective accorde à chacun des salariés qu'elle représente sans avoir à justifier d'une cession de créance de l'intéressé ». Pareille règle est contraire à celles relatives au mandat établi au Code civil du Québec et à celui qui échoit à l'avocat.

7 Parry Sound (district), Conseil d'administration des services sociaux C. S.E.E.F.P.O., section locale 324, [2003] 2 R.C.S. 157, 2003 CSC 42; Isidore Garon Itée c. Tremblay; Fillion et Frères (1976) inc. C. Syndicat national des employés de garage du Québec inc. [2006] 1 R.C.S. 27, 2006 CSC 2 [Isidore Garon]; Centre universitaire de santé McGill (Hôpital général de Montréal) c. Syndicat des employés de l'Hôpital général de Montréal, [2007] 1 R.C.S. 161, 2007 CSC 4, etc. II arrive même que des juges de la Cour suprême du Canada ne partagent pas entre eux le même entendement de leurs arrêts antérieurs !

8 Nous disons " notamment " parce que ce principe de la hiérarchie de normes est à la base même de l'ordre juridique établi dans une démocratie : constitution, loi, règlement, convention, contrat, etc. Le contrôle judiciaire de la constitutionnalité d'une loi, de la validité d'un règlement ou d'un contrat ne repose-t-il pas sur ce même principe de cohérence et qui exige le respect de l'ordre hiérarchique des normes ? Nous ne pouvons que déplorer, sans plus, le fait que la Cour suprême du Canada inversa cet ordre dans l'arrêt Isidore Garon, précité note 7. S'il nous fallait choisir entre cette approche, isolée il est vrai, et le libellé de l'article $62 \mathrm{Ct}$, nous devrions en conscience et en toute logique juridique nous en tenir à l'article $62 \mathrm{Ct}$ tant pour ce qui est formellement prescrit que pour les raisons qui justifient cet impératif.

9 C'est en ce sens que la formule dite de "l'incorporation » aux conventions collectives de ces règles de droit provenant des lois serait inexacte et parfois cause d'un possible dérapage tant en français qu'en droit. À notre avis, il n'y aurait pas d'incorporation, mais bien une superposition ordonnée et cohérente des règles conventionnelles à celles d'ordre public. II en est ainsi de la convention collective comme de tout autre contrat ou convention fondé juridiquement sur le Code civil et lui-même s'autorisant de la Constitution.

10 Par et en vertu de l'accréditation, un syndicat et un employeur acquièrent des droits et aussi des devoirs, lesquels sont circonscrits à ce même Code du travail!

11 Health, précité note 2 .

12 Combien de salariés s'interrogent maintenant à savoir si leur employeur et même leur syndicat recherchèrent vraiment un " accommodement raisonnable " suite à leur longue absence ou en raison de leur « handicap » ou de leur droit à l'exercice pratique d'une liberté fondamentale!

13 Depuis cette date, le contrat de travail n'est plus assimilé à un simple contrat de "location de services » comme s'il s'agissait de la location d'une simple quiddité ! Telle avait été la situation juridique formelle de 1865 à 1994 !

14 II n'empêche que si le syndicat n'a pas pris l'initiative de cette contestation de la légalité de la disposition conventionnelle, la tâche de l'arbitre devient plus délicate et suppose de sa part un certain degré de conviction personnelle et d'un savoir faire et dire pour réagir comme il se doit, mais sans plus!

15 Parce qu'il s'agit de l'administration de la justice, les règles générales rattachées à cet exercice doivent être respectées, notamment les règles relatives à l'équité procédurale et au droit de défense.

16 II est possible et nullement surprenant que certains arbitres n'aient pas une connaissance approfondie de chacun des arrêts de la Cour suprême du Canada ou qu'ils ne peuvent décliner de mémoire le libellé de l'article 234 LATMP ou 59.1 LNT, mais de telles qualités réelles ou apparentes ne sont pas des préalables essentiels pour aborder sagement et utilement une problématique complexe relevant des relations du travail. Une bonne connaissance 
de l'économie générale des relations du travail et des implications pratiques et juridiques possibles des conditions de travail pour le salarié et pour l'employeur importent davantage. Ainsi, cette même personne peut aussi fort bien saisir les données pertinentes de fait et de droit qu'apporteront les procureurs. Pour ceux qui doutent de la compétence de l'arbitre à se saisir de questions qui portent incidemment sur les chartes, voir l'arrêt Nouvelle-Écosse (Workers' Compensation Board) c. Martin; Nouvelle-Écosse (Workers' Compensation Board) c. Laseur, [2003] 2 R.C.S. 504, 2003 CSC 54.

17 Bisaillon c. Université Concordia, [2006] 1 R.C.S. 666, 2006 CSC 19 [Concordia] et notre commentaire in "Le Bulletin », C.A.Q., juillet 2007. II est vrai que de récentes décisions de la Cour d'appel n'aident guère à simplifier la question du partage des compétences jurisprudentielles : voir Gauthier c. Commission scolaire Marguerite-Bourgeoys, 2007 QCCA 1433 et Québec (Procureur général) c. Syndicat de la fonction publique du Québec, 2008 QCCA 1054.

18 On ne saurait inverser la situation juridique et s'interroger, en premier lieu, à savoir si la disposition de la loi est " compatible » à celle de la convention collective comme la Cour suprême du Canada sembla le faire dans I'arrêt Isidore Garon, précité note 7. Un tel procédé à rebours supposerait que la convention collective prévaudrait sur la loi, assertion qui, à notre avis, n'aurait aucun fondement historique, juridique et pratique.

19 Tel est notre entendement du droit sachant que bien des procureurs et des arbitres retiennent d'autres approches s'autorisant de la théorie de «l'incorporation » ou de la « compatibilité » comme nous l'avons déjà souligné.

20 Québec (Commission de l'industrie de la construction) c. C.T.C.U.M., [1986] 2 R.C.S. 327. Notre commentaire dans F. Morin, Jurisprudence commentée en droit du travail, Cowansville, Éditions Yvon Blais, 1992, 644 et s.

21 L'arbitre est certes désigné par les parties et, à défaut par le ministre du Travail mais sa fonction et ses pouvoirs lui proviennent notamment du Code du travail. La sentence arbitrale n'est-elle pas un acte public ayant force de " chose jugée »? Nous sommes alors hors du domaine strictement privé en ce qui a trait au processus arbitral et à celui qui a charge d'en présider le cours. La " servilité » même gantée au moyen d'excellents motifs demeure néanmoins ce qu'elle est! Dès le XVIe siècle, on s'intéressa à cette dernière question : voir Estienne de La Boétie, Discours de la servitude volontaire, Paris, Librairie philosophique J. Vrin, 2002.

22 À notre avis, ces dispositions du Code du travail permettent un renvoi certain à l'article 292 du Code de procédure civile qui autorise le président d'un tribunal à signaler aux procureurs les lacunes de la preuve afin qu'elles soient comblées. Avec respect pour les tenants d'une autre thèse, l'entente des procureurs ne pourrait valoir d'équivalent à une disposition conventionnelle ayant la portée fixée à l'article 67 Ct.

23 Nous le réitérons, le salarié est à la fois une personne distincte de sa collectivité et aussi membre de cette dernière. On ne peut, par simple voie métonymique, confondre l'un et l'autre, ni prendre l'un pour l'autre.

24 II est possible, en pareil contexte, que la prochaine nomination de cet arbitre par l'une de ces parties retarde quelque peu suite à cette "passe » ou impasse difficile. II ne s'agit alors que d'un risque inhérent à l'exercice de cette profession. 